\title{
Evaluación de la administración de carboximaltosa férrica intravenosa en la consulta de enfermedad renal crónica avanzada, para el control de la anemia
}

\author{
Esther García García, Mª Ángeles Romero González, Sonia Mendoza Mendoza, Alicia Gómez Gómez \\ Sección Nefrología. Hospital Universitario del Henares. Coslada. Madrid. España
}

\begin{abstract}
Resumen
La carboximaltosa férrica es una formulación de hierro endovenosa segura y de fácil dosificación, se puede administrar hasta 1 gramo iv en una sola sesión y permite ahorro de tiempo de los pacientes y de enfermería. Presentamos los resultados de su administración en una consulta de enfermedad renal crónica, analizando sus resultados y la repercusión en la carga de trabajo del personal de enfermería.
\end{abstract}

Material y Métodos: Se analizaron todos los pacientes en seguimiento de la consulta de enfermedad renal crónica en nuestro centro desde Ene-11 hasta Dic-14 que recibieron carboximaltosa férrica iv. Se registraron sus datos basales y los resultados clínicos y analíticos a los seis meses.

Resultados: Fueron identificados 85 pacientes en ese periodo. Edad media de $72 \pm 12$ años, con un filtrado glomerular estimado basal de $28 \pm 11 \mathrm{ml} / \mathrm{min}$ y a los seis meses de $30 \pm 11$ (p:n.s). La hemoglobina basal y a los seis meses fueron de $10 \pm 4$ y $11 \pm 3 \mathrm{~g} / \mathrm{dl}$ respectivamente $(\mathrm{p}:<0,001)$. El hematocrito basal y final fueron: $34 \pm 4$ vs $39 \pm 6 \%(p:<0,001)$. Los niveles de ferritina e índice de saturación de transferrina basales vs seis meses fueron respectivamente: $88 \pm 97$ vs $308 \pm 327 \mathrm{ng} /$ $\mathrm{ml}(\mathrm{p}:<0,001)$ y $11,2 \pm 6$ vs $22,3 \pm 11$ ( $p:<0,001)$. Dado que el tiempo medio de administración de cualquier formulación de hierro endovenoso es de 30 minutos, la estimación en base al número de administraciones es de un ahorro de 85 y 170 horas en estos 4 años si se compara con formulaciones que requieren de tres a

\section{Correspondencia:}

Esther García García

Sección Nefrología. Hospital Universitario del Henares

C/ Marie Curie, s/n. 28822 Coslada. Madrid

Email: esther.garcia@salud.madrid.org cinco sesiones respectivamente. A su vez el número de punciones se ha reducido, sin observarse complicaciones asociadas.

Conclusiones: La carboximaltosa férrica es segura y eficaz mostrando una recuperación de los niveles de hemoglobina y de los depósitos de hierro. Su facilidad de administración ha permitido ahorros sustanciales de tiempo. Esto junto a sus escasos efectos secundarios la hacen idónea para la administración ambulatoria.

\section{PALABRAS CLAVE}

- ANEMIA

- FERROPENIA

- PREDIÁLISIS

Evaluation of the intravenous administration of iron carboxymaltose for controlling anemia in advanced chronic kidney disease

\section{Abstract}

Ferric carboxymaltose is a safe and easy dosage of intravenous iron formulation; it can be administered intravenously up to $1 \mathrm{gram}$ in a single session and allows time savings of patients and nursing. We present the results of his administration in a unit of chronic kidney disease, analyzing the results and the impact on the workload of the nursing staff.

Materials and methods: All patients followed in the chronic kidney disease unit at our center from January 2011 to December 2014 and who received intravenous iron carboxymaltose were analyzed. Their baseline data and clinical and laboratory results at six months were recorded.

Results: 85 patients were identified during this period. Mean age of $72 \pm 12$ years, with a baseline 
estimated glomerular filtration rate of $28 \pm 11 \mathrm{ml} /$ $\min$ and at six months of $30 \pm 11(p>0.05)$. Baseline and at six months hemoglobin levels were $10 \pm 4$ and $11 \pm 3 \mathrm{~g} / \mathrm{dl}$, respectively $(\mathrm{p}<0.001)$. The basal and final hematocrit were: $34 \pm 4$ vs $39 \pm 6 \%(p<0.001)$. Baseline ferritin levels and transferrin saturation index versus levels at six months were respectively: $88 \pm 97$ vs $308 \pm 327 \mathrm{ng} / \mathrm{ml}(\mathrm{p}<0.001)$ and 11.2 \pm 6 vs $11 \pm 22.3(p<0.001)$. Since the average administration time by any formulation of intravenous iron is 30 minutes, the estimate based on the number of administrations is a saving of 85 and 170 hours on these four years when compared with formulations that require three to five respectively sessions. In turn, the number of punctures has been reduced without associated complications observed.

Conclusion: Carboxymaltose iron is safe and effective showing a recovery of hemoglobin levels and iron deposits. Its ease of administration has allowed substantial time savings. This, together with its few side effects makes it ideal for outpatient administration.

\section{KEYWORDS}

- ANEMIA

- IRON DEFICIENCY

- PREDIALYSIS

\section{Introducción}

La actividad de enfermería en las consultas de enfermedad renal crónica avanzada (ERCA) abarca numerosos aspectos y cada vez implica una mayor carga de trabajo. La anemia es un proceso muy frecuente en la enfermedad renal crónica que requiere una especial vigilancia ${ }^{1,2}$. La presencia de ferropenia es una de las habituales causas multifactoriales de dicha anemia y su control es un aspecto clave. Muchas formulaciones orales o no son bien toleradas o no alcanzan niveles aconsejados por lo que es necesario su administración intravenosa ${ }^{3,4}$. Esta práctica habitual en las consultas de ERCA, con las formulaciones clásicas, implica una dosis de prueba, varios días de administración iv, punciones repetidas, y por lo tanto mayor ocupación de la actividad de enfermería. La carboximaltosa férrica (CMF) es una formulación que permite una dosis alta de hierro en una sola administración iv, que posibilita reducir los tiempos de administración, las punciones y los transportes, así como optimizar la carga de trabajo del personal de enfermería, manteniendo una adecuada eficacia en el control de la anemia ${ }^{5,6,7 .}$. Mostramos nuestra experiencia en la consulta de ERCA en nuestro centro desde 2011 con el presente estudio, cuyo objetivo ha sido analizar la eficacia de la administración de CMF iv en el control de la anemia, así como valorar sus potenciales beneficios asociados respecto a la carga de trabajo del personal de enfermería.

\section{Material y Métodos}

Desde enero de 2011 se han estudiado de forma retrospectiva todos los pacientes en seguimiento con ERCA en nuestra consulta. Se han recogido los datos basales y a los seis meses de la administración de CMF, analizando los parámetros de anemia (hemoglobina, hematocrito, ferritina e índice de saturación de transferrina), los valores del filtrado glomerular, niveles de calcio, fósforo y PTH, así como los posibles efectos secundarios tras la administración de CMF.

De cara a analizar el consumo de tiempo de enfermería para conseguir los niveles de hierro deseados, se comparó el número de dosis necesarias con otras formulaciones, con la dosis única que se administró de CMF. Con las formulaciones previas eran necesarias de tres a cinco sesiones no consecutivas de administración iv. Cada sesión requería una punción venosa y su tiempo habitual era de 30-40 minutos.

La CMF es una formulación que permite hasta un gramo de administración de hierro iv en una sola toma, sin necesidad de dosis de carga. El tiempo de administración de $1 \mathrm{gr}$ iv de CMF (Ferinject ${ }^{\circledR}$, Vifor Pharma) se estableció en 30 minutos al igual que para administrar otras formulaciones de hierro intravenoso, aunque según la ficha técnica este tiempo podría ser menor. La dosis habitual administrada fue de $1000 \mathrm{mg}$, siempre que no se superara los $15 \mathrm{mg}$ por $\mathrm{Kg}$ de peso corporal, diluida en $250 \mathrm{cc}$ de solución de cloruro sódico al 0,9\%.

Todos los pacientes recibían el tratamiento el mismo día de la consulta tras valoración por el facultativo o como máximo al día siguiente, y se realizó en la sala de técnicas de enfermería, bajo supervisión continua de la enfermera. Para ello, se canalizaba una vía periférica mediante un catéter de pequeño calibre, intentando salvaguardar los accesos venosos del paciente.

Fueron excluidos pacientes con historial previo de reacciones a la administración de hierro iv. 
Análisis estadístico: Los datos analizados tienen una distribución normal, por lo que se utilizaron tests paramétricos. Los resultados se expresan en media con desviación Standard. Para la comparación de medias se ha utilizado el test de Student para datos apareados. Un valor de $p<$ de 0,05 fue considerado estadísticamente significativo.

\section{Resultados}

Se han tratado con CMF durante el periodo de estudio 85 pacientes, de los cuales 72 pacientes presentan los datos completos. Los datos basales se muestran en la tabla 1.

Tabla 1. Datos basales.

\begin{tabular}{|l|l|}
\hline Número de pacientes & 72 \\
\hline Varones/Mujeres & $30 / 42$ \\
\hline Edad Media (años) & $72 \pm 12$ \\
\hline Etiología enfermedad renal: & \\
\hline \multicolumn{1}{|c|}{ Diabetes Mellitus } & 20 \\
\hline \multicolumn{1}{|c|}{ Glomerulonefritis } & 11 \\
\hline \multicolumn{1}{|c|}{ Nefroangiosclerosis/Isquémica } & 22 \\
\hline \multicolumn{1}{|c|}{ Nefropatía Tubulo-Intersticial } & 8 \\
\hline \multicolumn{1}{|c|}{ No filiada } & 11 \\
\hline Datos Analíticos: & \\
\hline Hemoglobina (gr/dl) & $10,7 \pm 1,1$ \\
\hline Hematocrito (\%) & $33 \pm 4$ \\
\hline Ferritina (ng/ml) & $118 \pm 127$ \\
\hline IST (\%) & $13,4 \pm 5$ \\
\hline Creatinina (mg/dl) & $2,5 \pm 1$ \\
\hline FGE (ml/min, MDRD) & $26,4 \pm 11$ \\
\hline Calcio (mg/dl) & $9,1 \pm 0,5$ \\
\hline Fósforo (mg/dl) & $3,9 \pm 0,7$ \\
\hline PTH (pg/ml) & $169 \pm 108$ \\
\hline
\end{tabular}

IST: Índice de saturación de transferrina. FGE: Filtrado glomerular estimado.

La figura 1 y 2 muestra los resultados a de los niveles de hemoglobina, hematocrito y ferritina basales y a los 6 meses. La hemoglobina basal y a los seis meses fue de $10 \pm 4$ y $11 \pm 3 \mathrm{~g} / \mathrm{dl}$ respectivamente $(\mathrm{p}:<0,001)$. El hematocrito basal y final fueron: $34 \pm 4$ vs $39 \pm 6 \%$ ( $p:<0,001)$. Los niveles de ferritina e índice de saturación de transferrina basales y a los seis meses fueron respectivamente: $88 \pm 97$ vs $308 \pm 327 \mathrm{ng} / \mathrm{ml}(\mathrm{p}:<0,001)$ y $11,2 \pm 6$ vs $22,3 \pm 11$ ( $p:<0,001$ ). No hubo diferencias en el filtrado glomerular estimado, basal de $28 \pm 11 \mathrm{ml} /$ min y a los seis meses de $30 \pm 11$ (p:n.s). Tampoco se evi-

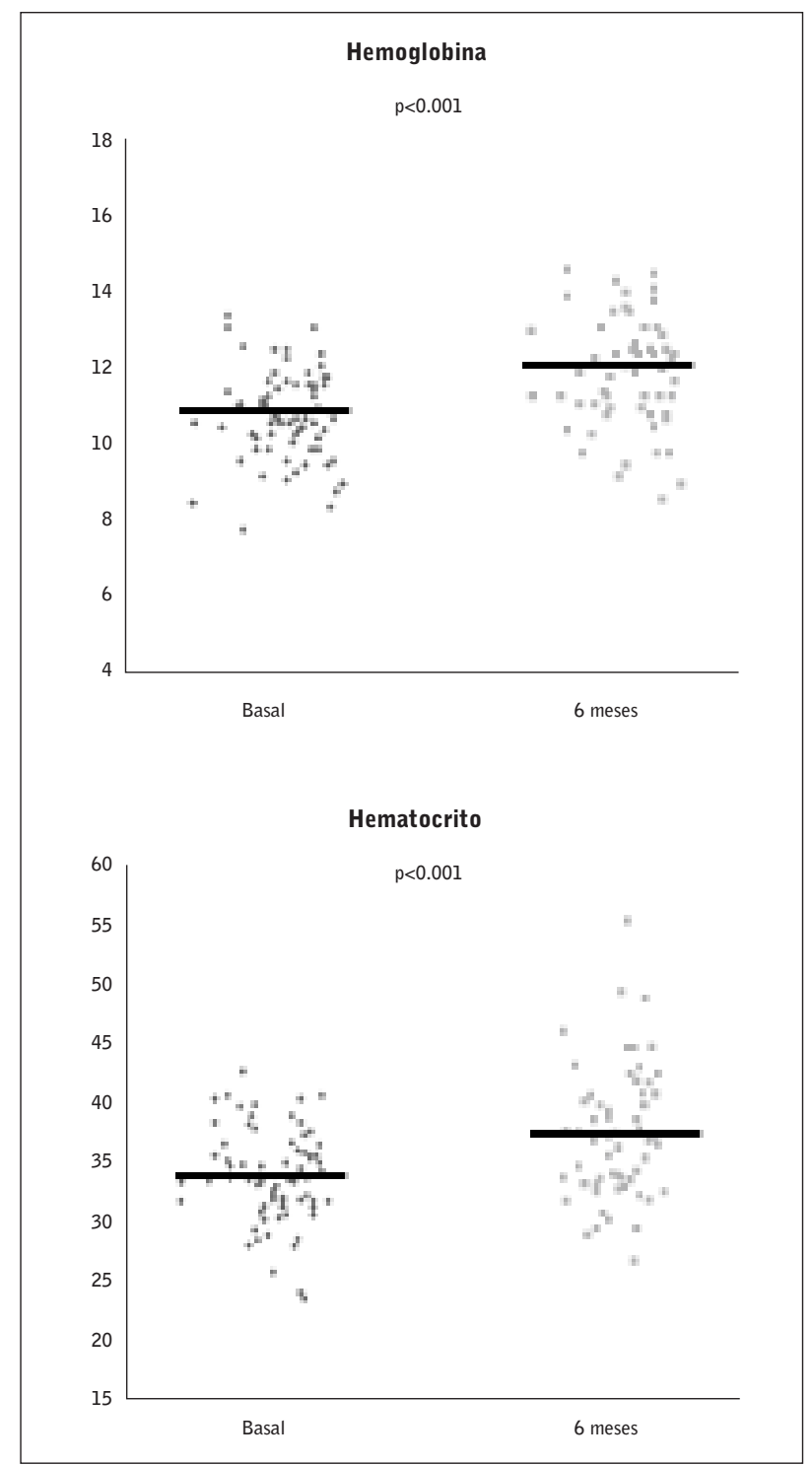

Figura 1. Niveles de hemoglobina y hematocrito basales y a los 6 meses.

denciaron diferencias entre los niveles de calcio y fósforo basales y a los seis meses.

Un $36 \%$ de los pacientes recibían tratamiento con factores estimulantes eritropoyéticos (FEE) en el momento de la administración de CMF iv, con una dosis media de $22 \mathrm{mcg} \pm 28$ de darbepoetina semanal. La dosis media de FEE a los seis meses fue de 19 mcg \pm 16 , estadísticamente no significativa.

El consumo de tiempo en la administración iv, en cualquier otra formulación de hierro endovenoso, habitualmente es de 30 minutos, siendo necesario entre tres y cinco sesiones en días diferentes para alcanzar la dosificación indicada. En base al número estimado de adminis- 


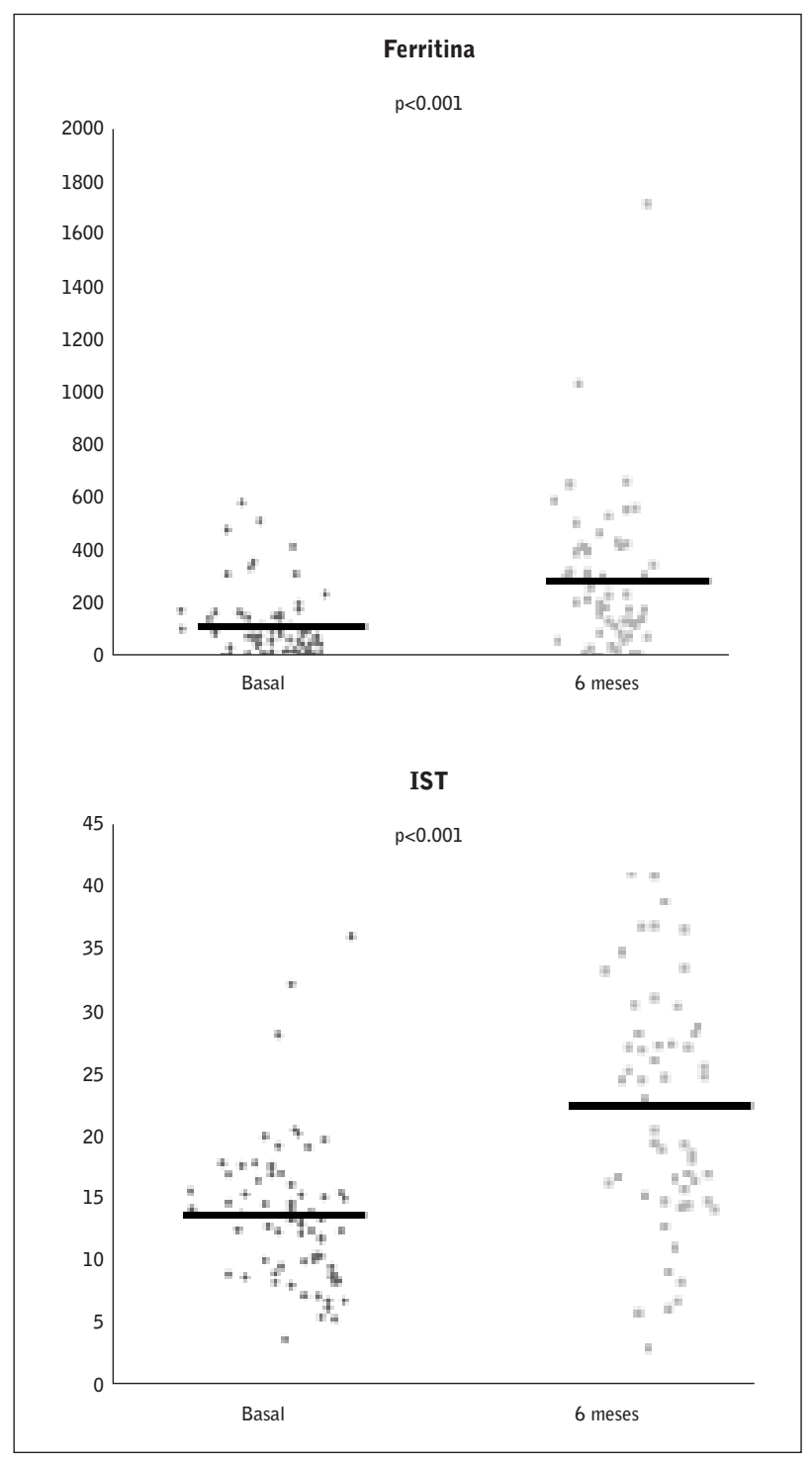

Figura 2. Niveles de ferritina e IST basales y a los 6 meses.

traciones, esta pauta supondría un ahorro de 72 y 144 horas en estos años, respectivamente, si se compara con las otras formulaciones de hierro iv. De este modo estimamos que permite reducir un $66 \%$ el número de punciones a los pacientes que sólo requieren una única dosis, comparado con los pacientes que requieren una pauta de 3 días, y hasta un $80 \%$, en pautas que requieren 5 días. No se han observado efectos secundarios destacables salvo tres episodios de hiperpigmentación cutánea por extravasación.

\section{Discusión}

La corrección de la anemia, especialmente en situación de déficit de hierro es un aspecto crucial para los enfermos con ERCA. La CMF en estudios previos ha demostrado eficacia en el control de la anemia, tanto en comparación con administraciones orales como iv. Los pacientes con ERCA están polimedicados y presentan una absorción oral comprometida por lo que las necesidades de tratamiento iv son frecuentes, estas formulaciones permiten un correcto tratamiento de la ferropenia ${ }^{8-9}$. Los trabajos previos de Qunibi y Charytan así lo han evidenciado y muestran resultado semejantes a nuestro estudio $0^{4,5}$.

Además la posología simplificada, que implica una disminución de consumo de tiempo tanto para el paciente como para el personal de enfermería, y su rapidez de repleción, confieren a la CMF un espectro idóneo para la administración ambulatoria. Este es un aspecto menos descrito en la literatura, aunque algunos trabajos como el de Bhandari o Clavet ya ponen de manifiesto los potenciales beneficios de esta formulación por encima del coste neto de un vial ${ }^{10,11}$.

En nuestro centro la administración de $1 \mathrm{gr}$ implica el uso de dos viales ( 1 vial $=96 €$ ). Aunque no disponemos de los costes detallados del material fungible, del coste del personal, de los tiempos de ocupación y de los traslados si son necesarios y además tenemos en cuenta la rapidez de acción en comparación con aquellas formulaciones que requieren al menos una semana para alcanzar niveles adecuados, el potencial beneficio puede ser mayor, a todos los niveles, por encima del coste neto de un gramo iv de CMF.

Las actuales guías de accesos vasculares en el paciente renal recomiendan preservar lo máximo posible el árbol vascular de este tipo de pacientes ${ }^{12}$. Asumiendo que con la administración de CMF sólo se requiere una punción, hemos conseguido reducir durante este tiempo, entre un 66 y un $80 \%$ el número total de punciones en estos pacientes, con el consiguiente beneficio para su red vascular.

Por último la reducción de la carga asistencial en el personal de enfermería, cada vez con mayor presión asistencial y recursos limitados debe ser un aspecto a tener en cuenta para incluir y disponer de la alternativa iv de la CMF. 


\section{Conclusiones}

La carboximaltosa férrica es eficaz en el control de la anemia, con escasos efectos secundarios. Su posología permite reducir el número de punciones, reducir el número de traslados y ahorrar tiempos de administración, por lo que la carga de trabajo puede reducirse permitiendo el desarrollo de otros aspectos también relevantes en la consulta de ERCA. Estas características la hacen idónea para la administración ambulatoria por encima de su coste neto en comparación con otras formulaciones.

Recibido: 7 octubre 15

Revisado: 12 noviembre 15

Modificado: 20 enero 16

Aceptado: 27 enero 16

\section{Bibliografía}

1. McClellan W, Aronoff SL, Bolton WK, Hood S, Lorber DL, Tang KL, et al. The prevalence of anemia in patients with chronic kidney disease. Curr Med Res Opin. 2004 Sep;20(9):1501-10.

2. Locatelli $F$, Nissenson $A R$, Barrett BJ, Walker RG, Wheeler DC, Eckardt KU, et al. Clinical practice guidelines for anemia in chronic kidney disease: problems and solutions. A position statement from Kidney Disease: Improving Global Outcomes (KDIG0). Kidney Int. 2008 Nov;74(10):1237-40.

3. Fishbane S, Pollack S, Feldman HI, Joffe M M. Iron indices in chronic kidney disease in the National Health and Nutritional Examination Survey 19882004. Clin J Am Soc Nephrol. 2009 Jan;4(1):5761.

4. Charytan C, Bernardo MV, Koch TA, Butcher $A$, Morris D, Bregman DB. Intravenous ferric carboxymaltose versus standard medical care in the treatment of iron deficiency anemia in patients with chronic kidney disease: a randomized, activecontrolled, multi-center study. Nephrol Dial Transplant. 2013 Apr;28(4):953-64.

5. Qunibi WY, Martínez C, Smith $M$, Benjamin J, Mangione A, Roger SD. A randomized controlled trial comparing intravenous ferric carboxymaltose with oral iron for treatment of iron deficiency anaemia of non-dialysis-dependent chronic kidney disease patients. Nephrol Dial Transplant. 2011 May;26(5):1599-607.

6. Covic A, Mircescu G The safety and efficacy of intravenous ferric carboxymaltose in anaemic patients undergoing haemodialysis: a multi-centre, open-label, clinical study. Nephrol Dial Transplant. 2010 Aug;25(8):2722-30.

7. Tagboto S, Cropper L, Turner J, Pugh-Clarke K. The efficacy of a single dose of intravenous ferric carboxymaltose (Ferinject) on anaemia in a predialysis population of chronic kidney disease patients. J Ren Care. 2009 Mar;35(1):18-23.

8. Szczech LA, Bregman DB, Harrington RA, Morris $D$, Butcher A, KochTA, et al. Randomized evaluation of efficacy and safety of ferric carboxymaltose in patients with iron deficiency anaemia and impaired renal function (REPAIR-IDA): rationale and study design. Nephrol Dial Transplant. 2010 Jul;25(7):2368-75.

9. Merino JL, Bueno B, Campos MA, Amézquita Y. Intravenous ferric carboxymaltose for the treatment of anemia in chronic renal disease. Rev Clin Esp. 2014 June - July;214(5):282-283.

10. Bhandari S. Update of a comparative analysis of cost minimization following the introduction of newly available intravenous iron therapies in hospital practice. Ther Clin Risk Manag. 2011;7:501-9.

11. Calvet $X$, Ruíz MÀ, Dosal A, Moreno L, López $M$, Figuerola $A$, et al. Cost-minimization analysis favours intravenous ferric carboxymaltose over ferric sucrose for the ambulatory treatment of severe iron deficiency. PLOS ONE 2012; 7(9): e45604.

12. Rodríguez JA, González E, Gutiérrez JM, et al. Guías de acceso vascular en hemodiálisis (Guías S.E.N.). Nefrología 2005;25(Supl 1):3-97. 